\title{
Clinico-Pathological Evaluation of Fever More Than Three Weeks : A Cross Sectional Study in A Tertiary Care Hospital
}

Md. Shameem Haidar ${ }^{1 *}$

'Department of Medicine Combined Military Hospital Dhaka, Bangladesh.
*Correspondence to:

Col (Dr.) Md. Shameem Haidar

Classified Specialist in Medicine

Combined Military Hospital

Dhaka Cantonment

Dhaka, Bangladesh

Mobile : +88 01769014614

E-mail: shameemhaidar@yahoo.com

www.banglajol.info/index.php/CMOSHMCJ

\begin{abstract}
Background: Fever is a common clinical presentation of a number of diseases. A sustained unexplained fever $>38.3^{\circ} \mathrm{C}$ lasting for $>3$ weeks without an established diagnosis despite intensive diagnostic evaluation is referred to as Fever of Unknown Origin (FUO). Fever more than three week remains a clinical challenge for physicians, as it may be attributed to a wide range of disorders, mainly infections, malignancies, non-infectious inflammatory diseases and miscellaneous diseases. The evaluation of the condition of a patient with fever of unknown origin requires a knowledge of those disorders that produce this syndrome, an awareness of the potential significance of subtle findings in the history and physical examination, and an appreciation of the value in this clinical setting of specific diagnostic procedures. In this report, we review these aspects of fever of unknown origin and outline a diagnostic approach to the persistently febrile patient. Objective: Purpose of this study was to clinico-pathological evaluation of fever more than three weekswith its aetiology and clinical spectrum. Methods: This cross-sectional study was conducted amongst adult males and females patients suffering from the fever of more than three weeks over period of two years at Combined Military Hospital, Chattogram Cantonment from January, 2016 to December, 2017. Sample was selected by purposive sampling technique. Inclusion criteria were $\mathrm{H} / \mathrm{O}$ fever or body temperature greater than $38.3^{\circ} \mathrm{C}$ on several occasions, accompanied by more than three weeks of illness and failure to reach a diagnosis after one week of inpatient investigation. Total 72 cases were enrolled according to selection criteria. Routine hematological, biochemical, imaging test were done and mid-stream urine samples were collected from these patients and subjected to culture. Detail demographic data were collected from the informant and recorded in structured case report form. Clinical examination and relevant investigation were done meticulously. Results: In this study age of participants at entry was $>20$ years, mean age was $38.04 \pm 11.08$. Female sex were significant number, sex ratio (F: M) was 1.25:1. Most common clinical presentations were persistent fever and generalized weakness (100.0\%), followed by arthralgia/ arthritis $(51.3 \%)$ anorexia $(44.4 \%)$ and headache $(34.7 \%)$. The focused fever of unknown origin diagnostic approach is based on hallmark clinical features characteristic of each disorder. Diagnostic significance of nonspecific clinical findings is enhanced when considered together. Of the infectious diseases that are associated with FUO, tuberculosis (Especially in extrapulmonary sites) was most common cause (eg. 13.8\%) and in malignant aetilogy, lymphoma was the major cause (eg. 11.1\%) of fever of unknown origin. Abdominal and or Pelvic abscesses (5.6\%) Colorectal carcinoma (5.6\%) Drug-induced fever (4.1\%) UTI (5.6\%) SLE (5.6\%) Rheumatoid arthritis (9.7\%) Dental abscesses (2.7\%) and Osteomyelitis (4.1\%) were the others common cause of fever of unknown origin. Conclusion: Fever is a common presenting complaint in hospital admitted patients. Most febrile illnesses
\end{abstract}


either resolve before a diagnosis can be made or develop distinguishing characteristics that lead to a clinical dilemma. Fever of Unknown Origin (FUO) is dynamic in its origin and will be an ongoing challenge to the clinician because of shifting disease epidemiology. In this study infection was predominant aetiology for febrile illness. Proper evaluation, rationale use of drugs and health awareness reduced the burden of Fever of unknown origin.

Key words: Fever more than three weeks; Fever of Unknown Origin (FUO); Pyrexia of Unknown Origin (PUO).

\section{INTRODUCTION}

Fever is a common clinical presentation of a number of diseases. A sustained unexplained fever $>38.3^{\circ} \mathrm{C}$ lasting for $>3$ weeks without an established diagnosis despite intensive diagnostic evaluation is referred to as Fever of Unknown Origin (FUO). FUO remains a clinical challenge for physicians, as it may be attributed to a wide range of disorders, mainly infections, malignancies, non-infectious inflammatory diseases and miscellaneous diseases ${ }^{1}$. The four categories of potential etiology of FUO are classic, nosocomial, immune deficient, and human immunodeficiency virus-related. The four subgroups of the differential diagnosis of FUO are infections, malignancies, autoimmune conditions, and miscellaneous. A thorough history, physical examination, and standard laboratory testing remain the basis of the initial evaluation of the patient with $\mathrm{FUO}^{2}$.

The term 'Fever of Unknown Origin' (FUO) was first introduced by Petersdorf and Beeson in 1961 based on an analysis of 100 cases. It is defined as: a temperature greater than $38.3^{\circ} \mathrm{C}$ on several occasions, accompanied by more than three weeks of illness and failure to reach a diagnosis after one week of inpatient investigation ${ }^{3}$. Several decades later, the criteria of FUO diagnosis have changed and it is currently defined by lack of a definitive diagnosis after appropriate inpatient or outpatient evaluation ${ }^{4}$. The etiologies of classic FUO mainly include infections, malignancies, non-infectious inflammatory diseases and miscellaneous causes, while some cases remain undiagnosed.

Fluctuations in body temperature of up to $2^{\circ} \mathrm{F}\left(1.1^{\circ} \mathrm{C}\right)$ are normal. Elevated body temperature can be physiologic, or caused by pathologic processes such as infection, inflammatory processes, or malignancy. The diagnosis occurs across all age groups and affects both sexes equally. However, age is an important factor in forming a differential diagnosis. Epidemiologic trends in the etiology of FUO vary throughout the world ${ }^{5}$. This timing allowed exclusion of patients with protracted but self-limited viral illnesses, giving time for studies to be completed. This has now been modified to include patients who are diagnosed after two outpatient visits or three days in hospital ${ }^{3}$.
The term 'Fever of Unknown Origin' (FUO) is also sometimes used. PUO and FUO are used interchangeably in the scientific literature. PUO is used throughout this article for the sake of consistency $^{3}$. Additional categories have now been added, including ${ }^{6,7}$. Nosocomial PUO in hospital patients with fever of $38.3^{\circ} \mathrm{C}$ on several occasions, caused by a process not present or incubating on admission, where initial cultures are negative and diagnosis unknown after three days of investigation. Neutropenic PUO, which includes patients with fever as above with $<1 \times 10^{9}$ neutrophils, with initial negative cultures and diagnosis uncertain after three days. HIV-associated PUO, which includes HIV-positive patients with fever as above for four weeks as outpatients or three days as inpatients, with an uncertain diagnosis after three days of investigation, where at least two days have been allowed for cultures to incubate ${ }^{3}$.

The four categories of potential etiology of FUO are centered on patient subtype - classic, nosocomial, immune deficient, and HIV-associated. Each group has a unique differential diagnosis based on characteristics and vulnerabilities and, therefore, a different process of evaluation ${ }^{2}$. The classic category includes patients who meet the original criteria of FUO, with a new emphasis on the ambulatory evaluation of these previously healthy patients ${ }^{8}$. The revised criteria require an evaluation of at least three days in the hospital, three outpatient visits, or one week of logical and intensive outpatient testing without clarification of the fever's cause ${ }^{9}$. The most common causes of classic FUO are infection, malignancy, and collagen vascular disease. Common bacterial causes are abscesses, tuberculosis, Urinary Tract Infections (UTIs) endocarditis, hepatobiliary infections, Osteomyelitis, etc ${ }^{3}$.

The focused fever of unknown origin diagnostic approach is based on hallmark clinical features characteristic of each disorder. Diagnostic significance of nonspecific clinical findings is enhanced when considered together ${ }^{10}$. In abscess there may be no localising symptoms. Risk factor includes- previous abdominal or pelvic surgery, trauma or history of diverticulosis or peritonitis increases the likelihood of an occult intra-abdominal abscess. They are most commonly in the subphrenic space, liver, right lower quadrant, retroperitoneal space or the pelvis in women. In tuberculosis - when dissemination has occurred (eg, in patients who are immunocompromised) the initial presentation is more likely to consist of constitutional symptoms than localising signs ${ }^{3}$.

Blood cultures are useful for bacteremic fevers of unknown origin, for example, brucellosis, typhoid/enteric fever, intravascular infections, and abscesses, but blood cultures are unnecessary and may be misleading for nonbacteremic infections, malignant/neoplastic, rheumatic/inflammatory, and miscellaneous fevers of unknown origin ${ }^{11,12}$. Urinary Tract Infections (UTIs) are rare cause of FUO. Perinephric abscesses occasionally fail to communicate with the urinary system, resulting in a normal urinalysis. Previous antibiotic therapy is the most frequent reason for negative blood cultures in PUO. Hepatobiliary infections (eg. cholangitis) can occur without 
local signs and with only mildly elevated or normal LFTs, especially in the elderly. Osteomyelitis - this usually causes localised pain or discomfort at least sporadically. Borrelia recurrentis - this is transmitted by ticks. It is responsible for causing relapsing fever. Other spirochetal diseases that can cause PUO these include Spirillum minor (Rat-bite fever) Borrelia burgdorferi (Lyme disease) and Treponema pallidum (Syphilis) ${ }^{3}$.

Neoplasmis another important causes for PUO. Because of a substantial increase in the elderly population, as well as advances in the diagnosis and treatment of diseases common in this population, malignancy has become a common etiologic consideration in elderly patients ${ }^{2}$. Hodgkin's lymphoma and nonHodgkin's lymphoma - these may cause PUO. Among solid tumours - renal cell carcinoma is most commonly associated with PUO. Solid tumours (Such as adenocarcinomas of the breast, liver, colon or pancreas) and liver metastases from any primary site - these may present with fever. Malignant histiocytosis this is a rare, rapidly progressive malignant disease. A wide variety of drugs can cause drug fever: The most common are betalactam antibiotics, procainamide (Now discontinued) and isoniazid. Stopping the drug generally leads to recovery within two days. It is usually accompanied by a rash $^{3}$.

Rheumatoid arthritis and rheumatic fever are inflammatory diseases that used to be commonly associated with FUO, but with advances in serologic testing, these conditions usually are diagnosed more promptly ${ }^{2}$. High-spiking fevers, non-pruritic rashes, arthralgias and myalgias, pharyngitis and lymphadenopathy typically are common manifestation. Polyarteritis Nodosa (PAN) rheumatoid arthritis, SLE, Still's diseaseand mixed connective tissue diseases also be considered. In granulomatous diseases, Sarcoidosis, Crohn's disease and Granulomatous hepatitis causes PUO. Giant cell arteritis and also the related polymyalgia rheumatica. Polyarteritis nodosa \& Behçet's disease has also been reported. Hyperthyroidism and subacute thyroiditis are the most common endocrine causes of PUO. Adrenal insufficiency is a rare but potentially fatal cause of $\mathrm{PUO}^{3}$. The initial approach to the patient presenting with fever should include a comprehensive history, physical examination, and appropriate laboratory testing. Newer diagnostic modalities, including updated serology, viral cultures, computed tomography, and magnetic resonance imaging, have important roles in the assessment of these patients ${ }^{2}$.

\section{MATERIALS AND METHODS}

This is cross-sectional study was conducted over a period of two year amongst the patients presented with temperature higher than $38.3^{\circ} \mathrm{C}$ on several occasions, a fever lasting more than three weeks; and a failure to reach a diagnosis despite one week of inpatient investigation. Total 72 patients included according to selection criteria. . Diagnosis was made on the basis of patient's statement, statement of the witness, characteristic features of manifestation, clinical examination and available medical records.
After fulfilling the inclusion and exclusion criteria, patient were enrolled with unique ID. Subjects were briefed about the objectives of the study, risk and benefits, freedom for participating in the study and confidentiality. Informed consent was obtained accordingly. Patient was managed according to the feature of corresponding aetiology. Careful attention to the skin, mucous membranes, and lymphatic system, as well as abdominal palpation for masses or organomegaly was conducted. The preliminary evaluation helps in the formulation of a differential diagnosis and guides further studies that are more invasive or expensive. These preliminary investigations include a complete blood count, liver function test, erythrocyte sedimentation rate, urinalysis, and basic cultures. The decision to obtain further diagnostic studies was based on abnormalities found in the initial laboratory work-up and not represent a haphazard use of costly or invasive modalities. Abdominal sonography, pelvic sonography, or CT scanning was performed early in the diagnostic process to rule out such common causes of FUO as intra-abdominal abscess or malignancy, depending on the primary evaluation. More invasive testing, such as lumbar puncture or biopsy of bone marrow, liver, or lymph nodes, was performed only when clinical suspicion shows that these tests are indicated or when the source of the fever remains unidentified after extensive evaluation. When the definitive diagnosis remains elusive and the complexity of the case increases, an infectious disease, rheumatology, or oncology consultation also taken.

All the information recorded in data sheet. Data was processed and analysed with the help of computer program Microsoft excel. Quantitative data was expressed as mean and standard deviation and qualitative data expressed as frequency and percentage. Comparison done by tabulation and graphical presentation in the form of tables, pie chart, graphs, bar diagrams, histogram \& charts etc.

\section{RESULTS}

The age of participants at entry was $>20$ years, mean age was $38.04 \pm 11.08$. Participants were randomly selected on male and female subject. Female sex were significant number, sex ratio (F: M) was 1.25:1.

Table 1 : Baseline characteristics of Study population $(n=72)$

\section{Characteristics with Indicator}

Age in yr $($ Mean $\pm \mathrm{SD})$

Sex ratio (F:M)

Occupation category (House wife)

Mean duration of illness (Day)

Major aetiology (Infection)

Major risk factors

Bacteria isolation (E.coli)

\section{Result} $38.04 \pm 11.08$

$28(49.12 \%)$ 
The median self-reported duration of questionnaire completion was 70 minutes (Range 50-90). Baseline clinical characteristics are: Occupation category (House wife) 29 (40.27\%) Duration of illness (Day) were $>27$ day, infection was the major cause of fever of unknown origin, noticed in 35(48.6\%) patients. Among the total 72 cases of patients, previous history of abdominal or pelvic surgery was in maximum patients, major bacteria isolation (E.coli) observed in 28(49.12\%) (Table 1).

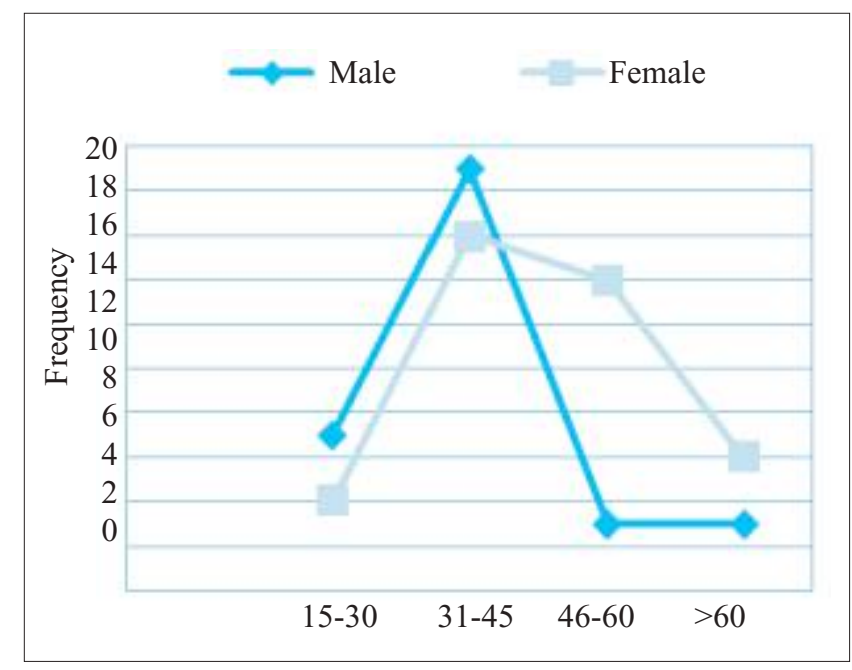

Figure 1 : Frequency of fever of unknown origin in age and sex variation $(\mathrm{n}=72)$

Frequency and susceptibility of pyrexia unknown origin commonly affected middle to elder age group. Age $>50$ disease is insignificant. In case of female 41-60 years was highest incidence and in case of male 31 to 45 years observed peak age for fever. (Figure-1).

Table 2 : Clinical symptoms of disease among respondents $(\mathrm{n}=72)$

$\begin{array}{lcc}\text { Manifestation } & \text { Number of Patients } & \text { Percentage } \\ \text { Fever }>38.5^{\circ} \mathrm{C} & 72 & 100.0 \\ \text { Generalized weakness } & 72 & 100.0 \\ \text { Arthralgia/ Arthritis } & 37 & 51.3 \\ \text { Anorexia } & 32 & 44.4 \\ \text { Headache } & 25 & 34.7 \\ \text { Abdominal pain } & 18 & 25.0 \\ \text { Oral ulceration } & 11 & 15.2 \\ \text { Myagia } & 15 & 20.8 \\ \text { Vomitting } & 7 & 9.7 \\ \text { Loss of body weight } & 15 & 20.8 \\ \text { Dysuria, frequency } & 8 & 11.1 \\ \text { Altered bowel habit } & 10 & 13.8\end{array}$

In this study most common clinical presentations of the patients with Pyrexia of Unknown Origin (PUO) were persistent fever and generalized weakness (100.0\%) followed by arthralgia/ arthritis (51.3\%) anorexia (44.4\%) and headache (34.7\%). Fever was acute onset $\&$ rises abruptly in some cases, reaching $39-40^{\circ} \mathrm{C}$, with chills and rigor. The joint symptom was arthralgia or arthritis, involvement was symmetric and often ankles wrists and small joints of the hand are the worst affected. Other symptoms were abdominal pain in $25.0 \%$, oral ulceration in $15.2 \%$, and loss of body weight in $20.8 \%$ of patients (Table 2).

On evaluation of laboratory profile, most of the patients $\mathrm{Hb} \%$ $(\mathrm{gm} / \mathrm{dl})$ was within normal range. Leucopenia $\left(<4 \times 10^{9} / \mathrm{L}\right)$ was detected in $56.9 \%$ of patients and thrombocytopenia $\left(<100 \times 10^{9} / \mathrm{L}\right)$ was in only 11 patients (Table 3$)$. In this series, blood culture was positive in $28(38.7 \%)$ of cases and negative in $44(61.1 \%)$ of cases.Among the Blood culture positive cases, maximum number of patients $21(75 \%)$ had gram negative organisms and only $7(25 \%)$ had gram positive organism. Among the blood culture positive, organism isolated mainly were gram negative, E coli $9(32.14 \%)$ \& Klebsiella pneumonia $7(25 \%)$ (Table 4$)$.

Table 3 : Common laboratory profile $(n=72)$

\begin{tabular}{lcc} 
Result & Number of Patients & Percentage \\
$\mathrm{Hb} \%(\mathrm{gm} / \mathrm{dl})$ mean $\pm \mathrm{SD}$ & mean \pm SD $(11.35 \pm 1.7)$ \\
Leucopenia $\left(<4 \times 10^{9} / \mathrm{L}\right)$ & 41 & 56.9 \\
Leucocytosis $\left(>4 \times 10^{9} / \mathrm{L}\right)$ & 18 & 25.0 \\
Thrombocytopenia & & \\
$\left(<100 \times 10^{9} / \mathrm{L}\right)$ & 11 & 15.2 \\
Significant CRP & 25 & 34.7 \\
IgG antibody & 18 & 25.0 \\
Triple antigen & 7 & 9.7 \\
\hline
\end{tabular}

Table 4 : Bacteriological Profile \& common isolated organism $(\mathrm{n}=72)$

\begin{tabular}{lcr} 
Organism & \multicolumn{1}{c}{ Frequency } & Percentage \\
Blood culture Positive & Gram (+) ve org. $7(25 \%)$ & $28(38.7 \%)$ \\
& Gram (-) ve org. 21 (75\%) & \\
Blood culture negative & & $44(61.1 \%)$ \\
Escherichia coli & 9 & 32.14 \\
Klebsiella pneumoniae & 7 & 25.0 \\
Staphylococcus aureus & 4 & 14.28 \\
Pseudomonas aeruginosa & 3 & 10.71 \\
Streptococcus spp. & 3 & 10.71 \\
Acinetobacter & 2 & 7.14 \\
\hline
\end{tabular}

Midstream urine samples were collected from patients into sterile container for urinalysis. Cultures with colony counts $\geq 10^{5}$ $\mathrm{cfu} / \mathrm{ml}$ were considered as significant bacteriuria. The organisms were identified using standard cultural, morphological and biochemical techniques. We found that $19(26.3 \%)$ of urine samples had significant bacteriuria (Figure 2). 


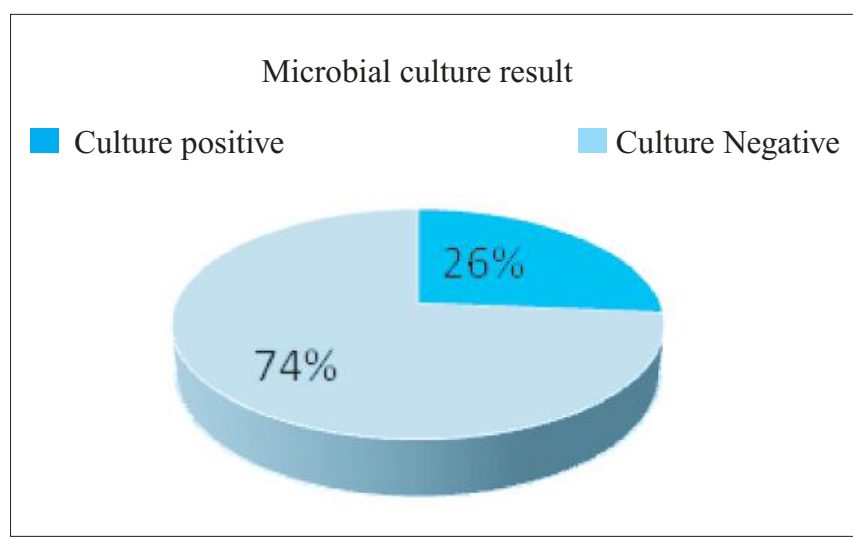

Figure 2 : Microbial culture result $(\mathrm{n}=72)$

Table 5 : Diagnosis of Fever of Unknown Origin $(n=72)$

\begin{tabular}{lrr} 
Diagnosis & Number of Patients & Percentage (\%) \\
Tuberculosis & & \\
(Especially & 10 & 13.8 \\
extrapulmonary) & 8 & 11.1 \\
Lymphoma & 7 & 9.7 \\
Rheumatoid arthritis & & \\
Abdominal and/or & 4 & 5.6 \\
pelvic abscesses & 4 & 5.6 \\
Colorectal carcinoma & & \\
Inflammatory bowel & 4 & 5.6 \\
disease & 4 & 5.6 \\
SLE & 4 & 5.6 \\
UTI & 3 & 4.1 \\
Liver abscess & 3 & 4.1 \\
Osteomyelitis & & \\
Hyperthyroidism and & 3 & 4.1 \\
subacute thyroiditis & 3 & 4.1 \\
Leukemia & 3 & 4.1 \\
Cholangitis & 3 & 4.1 \\
Drug-induced fever & 2 & 2.7 \\
Dental abscesses & 2 & 2.7 \\
Deep venous thrombosis & 2 & 2.7 \\
Still's disease & 2 & 2.7 \\
Septic thrombophlebitis & 1 & 1.3 \\
Reiter's syndrome & & \\
\hline
\end{tabular}

The focused fever of unknown origin diagnostic approach is based on hallmark clinical features characteristic of each disorder. Diagnostic significance of nonspecific clinical findings is enhanced when considered together. Of the infectious diseases that are associated with FUO, tuberculosis (Especially in extrapulmonary sites) lymphoma \& rheumatoid arthritis was the most common, detected in $13.8 \%, 11.1 \%$ \& $9.7 \%$ of patients respectively. SLE $(5.6 \%)$ colorectal carcinoma (5.6\%) Drug-induced fever (4.1\%) UTI (5.6\%) Leukemia (4.1\%) Dental abscesses (2.7\%) and Osteomyelitis (4.1\%) were the others common cause of fever of unknown origin (Table 5).

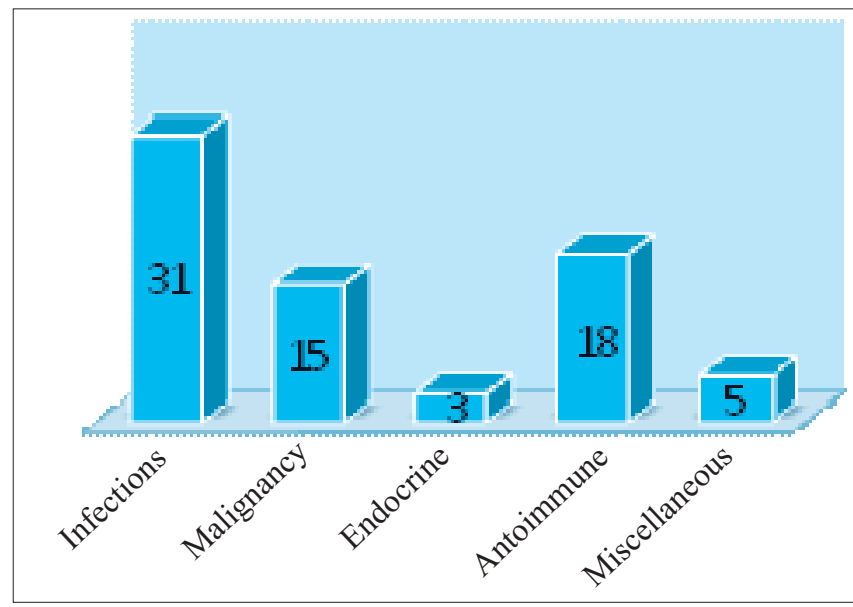

Figure 3 : Distribution of cases according to major aetiology $(n=72)$

The differential diagnosis of FUO generally is broken into five major subgroups: infections, malignancies, autoimmune conditions, endocrine disorder and miscellaneous. Infection was predominant aetiology of Fever of Unknown Origin, observed in 31 patients, malignancies noted in 15 patients, endocrine was 3 patients, autoimmune in 18 patients (Figure 3).

\section{DISCUSSION}

Our study design raises a number of important methodological issues, including patient selection, sample size and the prospective evaluation of frequency and aetiology of Fever of Unknown Origin, all of which may exert a powerful influence on the results. Fevers of unknown origin remain one of the most difficult diagnostic challenges in medicine. Because fever of unknown origin may be caused by over 200 malignant/ neoplastic, infectious, rheumatic/inflammatory, and miscellaneous disorders, clinicians often order non-clue-based imaging and specific testing early in the fever of unknown origin work-up, which may be inefficient/misleading ${ }^{10}$. Unlike most other fever-of-unknown-origin reviews, this article presents a clinical approach. Characteristic history and physical examination findings together with key nonspecific test abnormalities are the basis for a focused clue-directed fever of unknown origin work-up.

In this study age of participants at entry was $>20$ years, mean age was $38.04 \pm 11.08$. Participants were randomly selected on male and female subject. Female sex were significant number, sex ratio (F: M) was 1.25:1. Duration of illness (Day) were $>27$ day, infection was the major cause of fever of unknown origin, noticed in 35(48.6\%) patients. Among the total 72 cases of patients, previous history of abdominal or pelvic surgery was in maximum patients, major bacteria isolation (E.coli) observed in $28(49.12 \%)$.

FUO remains a clinical challenge for physicians, as the overall mortality from FUO is $12-35 \%$, with undiagnosed conditions 
accounting for $>20 \%$ in the 1990 s and 2000 s, despite the advances in diagnostic modalities ${ }^{13}$. Because FUOs are caused by a wide variety of disorders, the diagnostic approach to the FUO patient is often extensive consisting of three phases: at first initial evaluation should include relevant FUO history as well as physical examination that look particularly for diagnostic finding relevant to FUO. Initial nonspecific laboratory tests provide clues pointing toward a particular diagnosis while simultaneously eliminating other diagnosis. Second phase of FUO evaluation consists of a focused history and comprehensive physical examination with additional relevant lab tests. Third phase of FUO work-up is the definitive diagnostic testing including specific lab tests and biopsy to confirm the diagnosis 22 .

In this study most common clinical presentations were persistent fever and generalized weakness $(100.0 \%)$, followed by arthralgia/ arthritis (51.3\%) anorexia (44.4\%) and headache $(34.7 \%)$. Other symptoms wereabdominal pain in $25.0 \%$, oral ulceration in $15.2 \%$, and loss of body weight in $20.8 \%$ of patients. On evaluation of laboratory profile, most of the patients $\mathrm{Hb} \%$ (gm/dl) was within normal range. Leucopenia $(<4 \mathrm{x}$ $10^{9} / \mathrm{L}$ ) was detected in $56.9 \%$ of patients and thrombocytopenia $\left(<100 \times 10^{9} / \mathrm{L}\right)$ was in only 11 patientsBlood culture was positive in $28(38.7 \%)$ of cases and negative in $44(61.1 \%)$ of cases. Among the Blood culture positive cases, maximum number of patients $21(75 \%)$ had gram negative organisms and only $7(25 \%)$ had gram positive organism. Among the blood culture positive, organism isolated mainly were gram negative, E coli 9(32.14\%) \& Klebsiella pneumonia 7(25\%).

Findings consistent with result of other studies, eg. adult patients frequently present to the physician's with a fever (temperature higher than $\left.38.3^{\circ} \mathrm{C}\left[100.9^{\circ} \mathrm{F}\right]\right)^{2}$. Most febrile conditions are readily diagnosed on the basis of presenting symptoms and a problem-focused physical examination. Occasionally, simple testing such as a complete blood count or urine culture is required to make a definitive diagnosis. Viral illnesses (eg. upper respiratory infections) account for most of these self-limiting cases and usually resolve within two weeks ${ }^{2}$. When fever persists, a more extensive diagnostic investigation should be conducted. Although some persistent fevers are manifestations of serious illnesses, most can be readily diagnosed and treated.

Each fever of unknown origin category has clinical hallmarks, for example, usually, malignant/neoplastic disorders are associated with early anorexia and significant weight loss. With infectious fevers of unknown origin, chills are common, but weight loss less pronounced and anorexia late. Excluding vasculitis, synovitis is the rheumatic/inflammatory hallmark. While hallmark features suggest particular fever of unknown origin categories, some findings essentially eliminate a fever of unknown-origin category, for example, rigors eliminate the rheumatic/inflammatory category of fever ${ }^{10,14}$. The most diagnostically difficult fevers of unknown origin have no localizing signs ${ }^{10}$.
Blood cultures are useful for bacteremic fevers of unknown origin, for example, brucellosis, typhoid/enteric fever, intravascular infections, and abscesses, but blood cultures are unnecessary and may be misleading for nonbacteremic infections, malignant/neoplastic, rheumatic/inflammatory, and miscellaneous fevers of unknown origin ${ }^{10}$.

The focused fever of unknown origin diagnostic approach is based on hallmark clinical features characteristic of each disorder. Diagnostic significance of nonspecific clinical findings is enhanced when considered together. Of the infectious diseases that are associated with FUO, tuberculosis (Especially in extrapulmonary sites) lymphoma \& rheumatoid arthritis was the most common, detected in $13.8 \%, 11.1 \%$ \& $9.7 \%$ of patients respectively. SLE $(5.6 \%)$ colorectal carcinoma (5.6\%) Drug-induced fever (4.1\%), UTI (5.6\%), Leukemia (4.1\%), Dental abscesses (2.7\%) and Osteomyelitis (4.1\%) were the others common cause of fever of unknown origin.

Literature review suggested that common differential diagnoses of pyrexia of unknown origin - infective, eg. bacterial: pulmonary or extrapulmonary $\mathrm{TB}$, pneumonia, urinary tract infection, pelvic abscess, endocarditis, etc. Viral causes are gastroenteritis, hepatitis, HIV, EBV, CMV. Inflammatory causes are vasculitides (e.g. SLE), rheumatoid arthritis, crohn's disease. Malignancy include lymphomas, myeloma, any, especially if disseminated malignancy ${ }^{15}$. Another study demonstrated that most cases are unusual presentations of common diseases eg, tuberculosis, endocarditis, gallbladder disease and HIV infection, rather than rare or exotic diseases ${ }^{16}$. In adults, infections and cancer (25-40\% of cases each) account for most PUOs ${ }^{17}$. Autoimmune disorders account for $10-20 \%$ of cases ${ }^{18}$.

FUO is a common manifestation of a number of diseases, which are classified into infections, malignancies, noninfectious inflammatory diseases, miscellaneous causes and undiagnosed conditions ${ }^{13}$. Among these etiologies, infection was the most common cause of FUO in the 1961 survey ${ }^{19}$. However, according to Petersdorf's study published in 1992, neoplastic disease had surpassed infectious diseases as the etiology of classic $\mathrm{FUO}^{4}$, while in more recent studies, the percentage of neoplastic causes has decreased and that of noninfectious inflammatory diseases and undiagnosed conditions has increased ${ }^{13}$. The changes of the etiology proportion of FUO may be attributed to diagnostic advances, particularly the improvement of imaging and microbiological studies. Among the neoplastic causes of FUO, malignant lymphomas are the most common ${ }^{1}$.

In this study the differential diagnosis of FUO generally is broken into five major subgroups: infections, malignancies, autoimmune conditions, endocrine disorder and miscellaneous. Infection was predominant aetiology of Fever of Unknown Origin (FUO) observed in 31 patients; malignancies noted in 15 patients, endocrine was 3 patients, autoimmune in 18 patients. 
In a study reported that the final diagnoses of 31 cases of fever of unknown origin (FUO) and their distribution in the respective diagnostic categories were: infections $32 \%(10 / 31)$, non-infectious inflammatory disease $55 \%$ (17/31), and malignancy $13 \%$ (4/31). CT scan successfully identified an infectious, inflammatory, or neoplastic cause of fever in 10 of the 22 patients $(45 \%)$ who underwent this scan ${ }^{20}$. There are few other clinical presentations that produce such a wide array of differential diagnoses, including infections, malignancy and connective tissue disorders. Due to the high mortality rate of many of these conditions, especially if diagnosis is delayed, a high degree of suspicion is imperative. Thorough clinical assessment is vital to provide diagnostic clues and tailor investigations $^{21}$

\section{CONCLUSION}

Pyrexia of unknown origin has a wide range of differential diagnoses including infections, malignancies and connective tissue disorders, so requires prompt and appropriate investigations. One of the problems most frequently encountered in medical practice is the diagnosis of prolonged fever with or without local signs of disease. This problem perplexes both the physician and the patient and is labeled as FUO. The definition, classification and clinical approach, diagnosis and treatment have been discussed. It is important to realize FUO may represent uncommon manifestation of common disease. Hence the work-up should be cost effective and thoughtful and clinically appropriate. Empirical treatment sometimes may be justified, however one should remember that treatment should not be worse than disease. In India infections notably extra pulmonary tuberculosis is the most common cause of FUO. Noninfectious causes like collagen vascular disease and neoplasms are becoming important differential diagnosis.

\section{DISCLOSURE}

The author declared no competing interest. 


\section{REFERENCES}

1. Sun P, Cheng B, Wang J, He P. Fever of unknown origin revealed to be primary splenic lymphoma: A rare case report with review of the literature. Molecular \& Clinical Oncology. 2017; 6(2): 177-181.

2. Roth L and Basello G. Approach to the Adult Patient with Fever of Unknown Origin. Am Fam Physician. 2003;68(11):2223-2229.

3. Knott L. Pyrexia of Unknown Origin. 19 Oct 2016. Downloaded from: https://patient.info/doctor/pyrexia-of-unknown-origin. Retrieved on January 2018.

4. Petersdorf RG: Fever of unknown origin. An old friend revisited. Arch Intern Med. 1992;152:21-22

5. Roth A. Evaluation of fever of unknown origin in adults. Downloaded from: https://bestpractice.bmj.com/topics/en-us/375. Retrieved on January 2018.

6. Petersdorf RG, Beeson PB. Fever of unexplained origin: Report on 100 cases. Medicine (Baltimore). 1961; 40:1-30.

7. Hayakawa K, Ramasamy B, Chandrasekar PH. Fever of unknown origin: An evidence-based review. Am J Med Sci. 2012;344(4): 307-316.

8. Durack DT. Fever of unknown origin. In: Mackowiak PA, ed. Fever: Basic mechanisms and management. 2d ed. Philadelphia: LippincottRaven. 1997;237-249.

9. Durack DT, Street AC. Fever of unknown origin: Reexamined and redefined. Curr Clin Top Infect Dis. 1991;11:35-51.

10. Cunha B, Lortholary O, Cunha C. Fever of Unknown Origin: A Clinical Approach. The American Journal of Medicine. 2015;128:1138.e1-1138.e15.

11. Cunha CB. Prolonged and perplexing fevers in antiquity: Malaria and typhoid fever. Infect Dis Clin North Am. 2007;21:857-866.

12. Cunha BA. Fever of unknown origin: Clinical overview and perspective. In: Cunha BA, ed.Fever of Unknown Origin. New York: Informa Healthcare. 2007;1-8.

13. Mourad O, Palda V and Detsky AS: A comprehensive evidence-based approach to fever of unknown origin. Arch Intern Med. 2003; 163:545-551.

14. Cunha BA. Fever of unknown origin.Infect Dis Clin North Am. 1996; 10: 111-128.

15. Differential Diagnosis of Pyrexia of Unknown Origin (PUO). Oxford Medical Education. 2008.

16. PUO in late stage HIV: A system based approach; British HIV Association (BHIVA). 2009.

17. Mansueto P, Di Lorenzo G, Rizzo M, et al. Fever of unknown origin in a Mediterranean survey from a division of internal medicine: Report of 91 cases during a 12-year-period (1991-2002). Intern Emerg Med. 2008.

18. Ergonul O, Willke A, Azap A et al. Revised definition of 'fever of unknown origin': limitations and opportunities. J Infect. 2005; 50(1):1-5.

19. Petersdorf RG and Beeson PB: Fever of unexplained origin: Report on 100 cases. Medicine (Baltimore). 1961;40:1-30.

20. Pedersen TI, Roed C, Knudsen LS, Loft A, Skinhoj P \& Dam Nielsen SD. Fever of unknown origin: A retrospective study of 52 cases with evaluation of the diagnostic utility of FDG-PET/CT. Scandinavian Journal of Infectious Disease. 2012; 44(1):18-23.

21. Oswald A. Pyrexia of Unknown Origin - Differential Diagnosis? Res Medica. 2000;21(1):1-6.

22. Shantaram V, Narendra AMVR. Approach to the Patient with Fever of Unknown Origin. 2000;11:44-49. 